\title{
Supplementary Data of
}

\section{A Mechanistic Study of a Potent and Selective Epidermal Growth Factor Receptor Inhibitor against the L858R/T790M Resistance Mutation}

\author{
Farideh Badichi Akher, ${ }^{*} \dagger, \ddagger$ Abdolkarim Farrokhzadeh, ${ }^{\S}$ Neil Ravenscroft, ${ }^{\ddagger}$ and Michelle M. \\ Kuttel $^{*}, \dagger$ \\ ${ }^{\dagger}$ Department of Computer Science, University of Cape Town, Cape Town 7701, South Africa \\ tDepartment of Chemistry, University of Cape Town, Cape Town 7701, South Africa \\ ${ }^{\S}$ School of Chemistry and Physics, University of KwaZulu-Natal, Private Bag X01, \\ Pietermaritzburg 3209, South Africa \\ *Corresponding Authors: Farideh Badichi Akher, Michelle M. Kuttel \\ Email addresses: farideh.badichiakher@uct.ac.za, mkuttel@cs.uct.ac.za
}

Telephone: +27 (0) 844218946

Figure S1. Time series plots of RMSD for $\mathrm{C} \alpha$ atoms (angstroms) with respect to the initial structure for the $\mathrm{EGFR}^{\mathrm{WT}-\mathrm{APO}}$ (magenta), EGFR ${ }^{\mathrm{DM}-\mathrm{APO}}$ (green), EGFR ${ }^{\mathrm{WT}}-\mathbf{2 0 g}$ (black), and EGFR $^{\text {DM }}-20 g$ (red) complexes. (A) Whole structure C $\alpha$ RMSD plot of the simulated systems. (B) Post-equilibrated whole structure $\mathrm{C} \alpha$ RMSD plot from 200 to 250 ns indicating the degree of structural instability at these periods.

Figure S2. Dynamical effects of the T790M/L858R double mutant on protein backbone architecture. Trajectorial root-mean-square fluctuation (RMSF) estimations for (A) EGFR WT-APO (magenta) and EGFR ${ }^{\mathrm{WT}}-\mathbf{2 0 g}$ (black), (B) EGFR ${ }^{\mathrm{DM}-\mathrm{APO}}$ (green) and EGFR ${ }^{\mathrm{DM}}-\mathbf{2 0 g}$ (red), and (C) $E G^{W T}-20 g$ (black) and EGFR $^{\text {DM }}-20 g$ (red). Highly fluctuating EGFR regions are correspondingly numbered. (D) Structure of EGFR ${ }^{\mathrm{WT}}-\mathbf{2 0 g}$ showing labeled regions with large residual (RMSF) fluctuations.

Table S1. Table S1. Binding Free Energies and Energy Contributions of $20 \mathrm{~g}$ in complex with $\mathrm{EGFR}^{\mathrm{WT}}$ and $\mathrm{EGFR}^{\mathrm{DM}}$ (kilocalories per mole). 

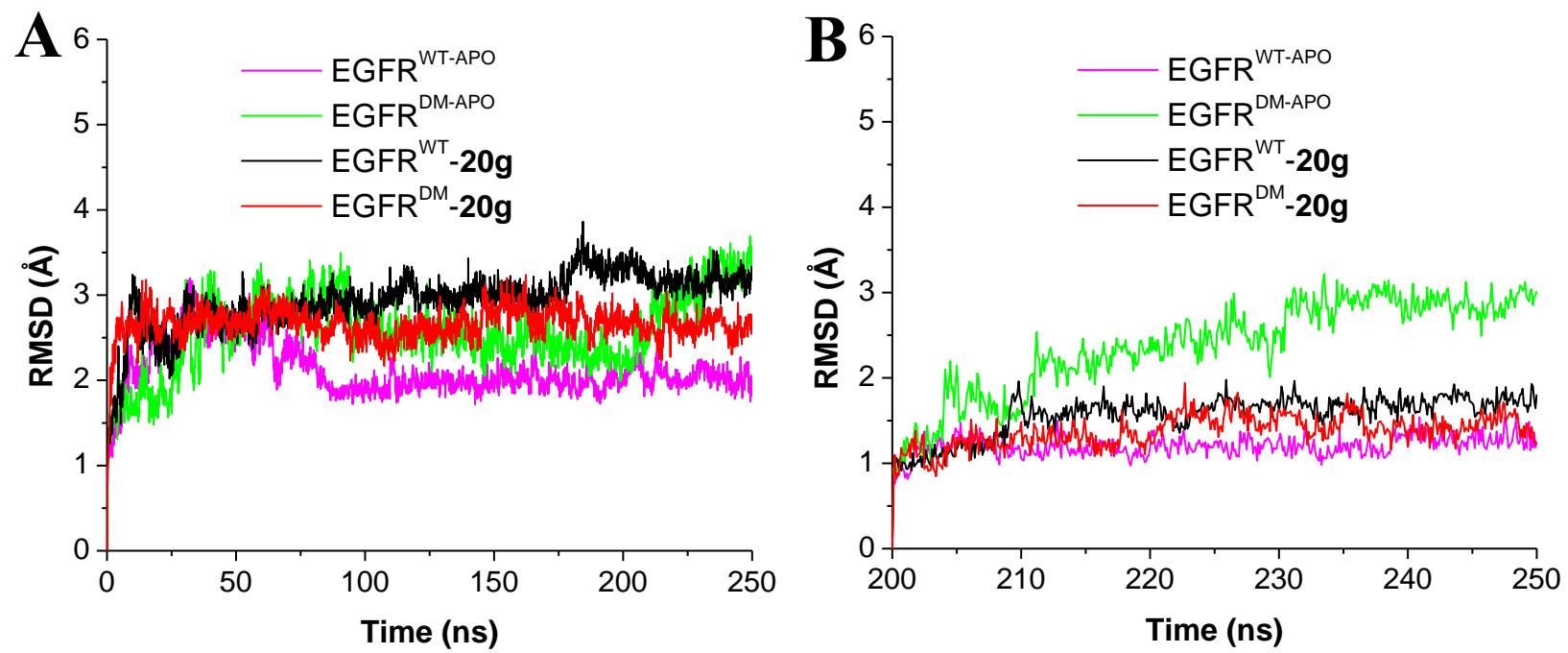

Figure S1. Time series plots of RMSD for $\mathrm{C} \alpha$ atoms (angstroms) with respect to the initial structure for the EGFR ${ }^{\mathrm{WT}-\mathrm{APO}}$ (magenta), EGFR ${ }^{\mathrm{DM}-\mathrm{APO}}$ (green), EGFR ${ }^{\mathrm{WT}}-\mathbf{2 0 g}$ (black), and EGFR $^{\text {DM }}-20 g$ (red) complexes. (A) Whole structure C $\alpha$ RMSD plot of the simulated systems. (B) Post-equilibrated whole structure $\mathrm{C} \alpha$ RMSD plot from 200 to 250 ns indicating the degree of structural instability at these periods. 

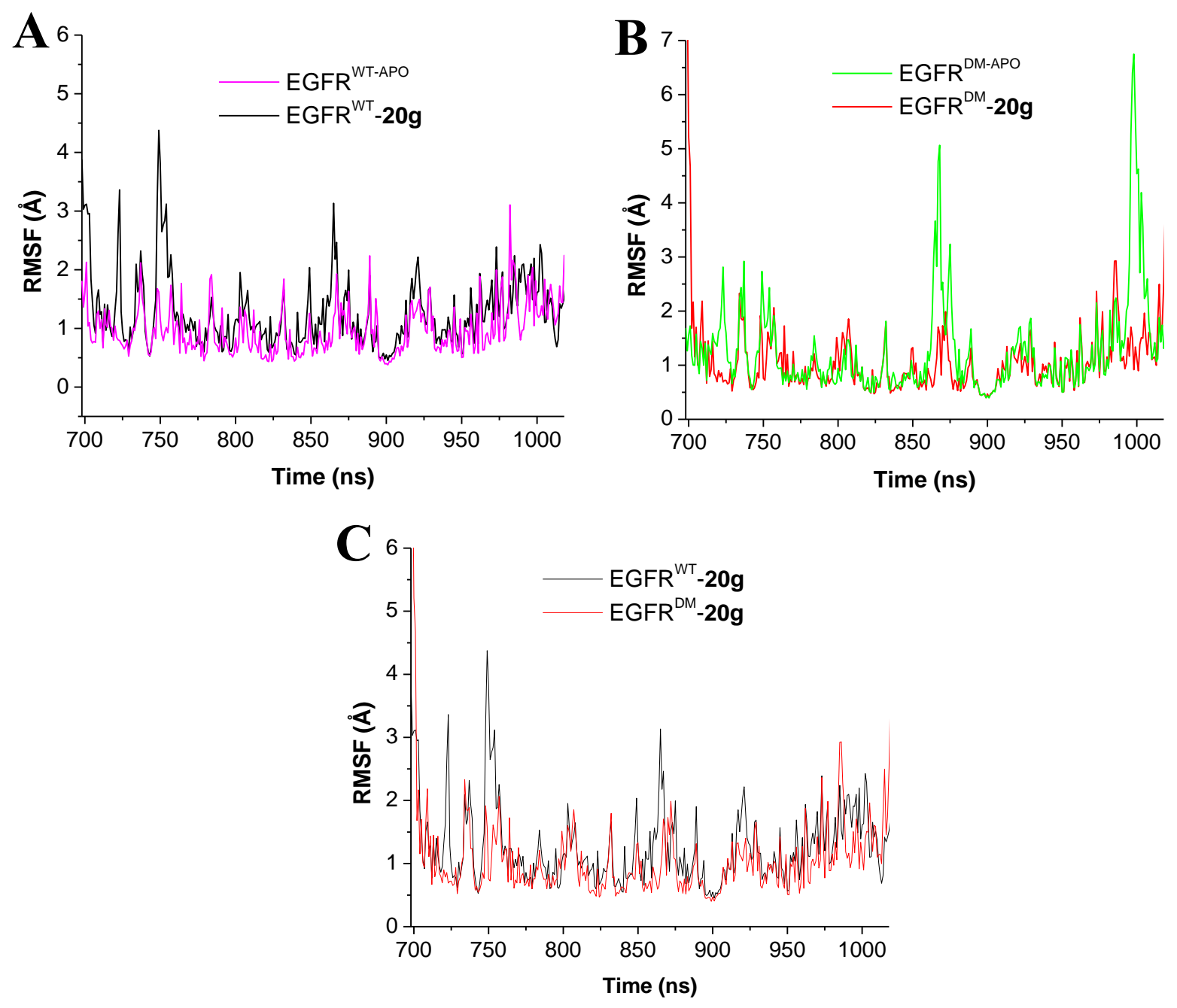

Figure S2. Dynamical effects of the T790M/L858R double mutant on protein backbone architecture. Trajectorial root-mean-square fluctuation (RMSF) estimations for (A) EGFR WT-APO (magenta) and EGFR ${ }^{\mathrm{WT}}-\mathbf{2 0 g}$ (black), (B) EGFR ${ }^{\mathrm{DM}-\mathrm{APO}}$ (green) and EGFR ${ }^{\mathrm{DM}}-\mathbf{2 0 g}$ (red), and (C) $E^{E G F R}{ }^{W T}-20 g$ (black) and $E^{2} F^{D M}-20 g$ (red). Highly fluctuating EGFR regions are correspondingly numbered. (D) Structure of EGFR ${ }^{\mathrm{WT}}-\mathbf{2 0 g}$ showing labeled regions with large residual (RMSF) fluctuations. 
Table S1. Binding Free Energies and Energy Contributions of $20 \mathrm{~g}$ in complex with EGFR ${ }^{\mathrm{WT}}$ and $\mathrm{EGFR}^{\mathrm{DM}}$ (kilocalories per mole).

\begin{tabular}{ccc}
\hline & \multicolumn{2}{c}{ Complexes } \\
\cline { 2 - 3 } Energy & EGFR $^{\mathrm{WT}}-\mathbf{2 0 g}$ & EGFR $^{\mathrm{DM}}-\mathbf{2 0 g}$ \\
\hline$\Delta E_{\mathrm{vdW}}$ & $-51.93( \pm 0.11)$ & $-52.84( \pm 0.10)$ \\
$\Delta E_{\text {ele }}$ & $-20.45( \pm 0.21)$ & $-14.77( \pm 0.37)$ \\
$\Delta G_{\text {gas }}$ & $-72.38( \pm 0.16)$ & $-67.61( \pm 0.24)$ \\
$\Delta G_{\text {ele,sol(GB) }}$ & $37.78( \pm 0.15)$ & $30.99( \pm 0.31)$ \\
$\Delta G_{\text {np,sol }}$ & $-7.58( \pm 0.24)$ & $-7.29( \pm 0.36)$ \\
$\Delta G_{\text {sol }}$ & $30.20( \pm 0.20)$ & $23.70( \pm 0.34)$ \\
$\Delta H$ & $-42.18( \pm 0.18)$ & $-43.91( \pm 0.29)$ \\
$T \Delta S$ & $-0.01( \pm 0.16)$ & $0.99( \pm 0.20)$ \\
$\Delta G_{\text {bind }}$ & $-42.17( \pm 0.14)$ & $-44.90( \pm 0.11)$ \\
$I C 50$ & $79.00( \pm 14.00)$ & $0.30( \pm 0.20)$ \\
\hline
\end{tabular}

